\title{
Design of Polymer Networks Involving a Photoinduced Electronic Transmission Circuit toward Artificial Photosynthesis
}

\author{
Kosuke Okeyoshi ${ }^{1}$, Ryuzo Kawamura ${ }^{2}$, Ryo Yoshida ${ }^{3}$, and Yoshihito Osada ${ }^{4, *}$ \\ ${ }^{1}$ School of Materials Science, Japan Advanced Institute of Science and Technology \\ 1-1 Asahidai, Nomi-shi, Ishikawa 923-1292, Japan \\ ${ }^{2}$ Department of Chemistry, Faculty of Science, Saitama University \\ 225 Shimo-okubo, Sakura-ku, Saitama 338-8570, Japan \\ ${ }^{3}$ Department of Materials Engineering, Graduate School of Engineering, The University of \\ Tokyo \\ 7-3-1 Hongo, Bunkyo-ku, Tokyo 113-8656, Japan \\ ${ }^{4}$ RIKEN \\ 2-1 Hirosawa, Wako-shi, Saitama 351-0198, Japan \\ FAX: +81-48-467-9300; TEL: +81-48-467-2816 \\ osadayoshi@riken.jp
}


Table S1. Molecular weight and molar ratio of $\mathrm{Ru}(\mathrm{bpy}){ }_{3}{ }^{2+}$ unit in polymers.

\begin{tabular}{l|cccc}
\hline & Mw & Mn & Mw/Mn & $\begin{array}{c}\text { molar ratio of } \\
\mathrm{Ru}(\mathrm{bpy})_{3}{ }^{2+} \text { unit }^{c)}\end{array}$ \\
\hline poly(NIPAAm-co-Ru(bpy) $\left.)_{3}\right)$ & $97,000^{a)}$ & $35,000^{a)}$ & $2.8^{a)}$ & $0.75 \mathrm{~mol} \%$ \\
poly(AAm-co-Ru(bpy) $\left.)_{3}\right)$ & $30,000^{b)}$ & $15,000^{b)}$ & $2.0^{b)}$ & $0.46 \mathrm{~mol} \%$ \\
\hline
\end{tabular}

${ }^{a}$ Determined by GPC (eluting solution: methanol + $10 \mathrm{mM} \mathrm{LiBr}, \mathrm{PEO}$ ).

${ }^{b}$ Determined by GPC (eluting solution: $50 \mathrm{mM}$ phosphate buffer, pH6.9, PEO).

${ }^{c}$ Determined by the absorption peak strength of polymer solution $(\lambda=460 \mathrm{~nm})$. The initial concentration of the $\mathrm{Ru}(\mathrm{bpy})_{3}{ }^{2+}$ for polymerization was $2.0 \mathrm{~mol} \%$. 
a

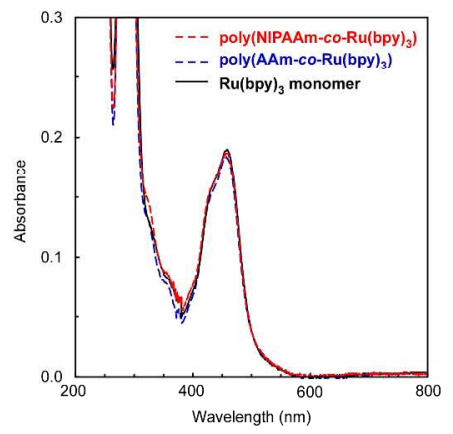

C

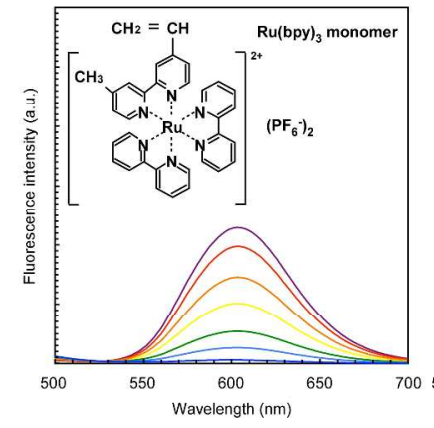

b

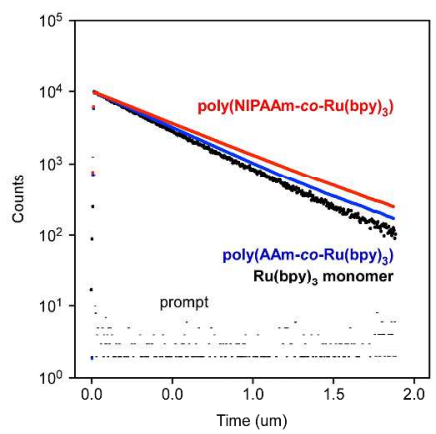

Figure S1. a) Absorption spectra of the $\mathrm{Ru}(\mathrm{bpy})_{3}{ }^{2+}$ monomer solution, poly(AAm-co-Ru(bpy) $\left.)_{3}\right)$ solution, and poly(NIPAAm-co-Ru(bpy) ${ }_{3}$ ) solution. b) Logarithmic plot of emission decay of the solutions. c) Fluorescence spectra at each concentration of $\mathrm{Ru}(\mathrm{bpy})_{3}{ }^{2+}$ in each solutions. d) Relation between absorption peak strength at $460 \mathrm{~nm}$ and fluorescence peak intensity for each solution. $[\mathrm{EDTA}]_{0}=50 \mathrm{mM}$.

Table S2 Luminescence life time observed for the solutions.

\begin{tabular}{lcccc}
\hline \multicolumn{1}{c}{ Solution } & $B_{1}$ & $\tau_{1}(\mathrm{~ms})$ & $B_{2}$ & $\tau_{2}(\mathrm{~ns})$ \\
\hline $\mathrm{Ru}(\mathrm{bpy})_{3}$ monomer & $-5.1 \times 10^{-3}$ & 1.72 & $8.3 \times 10^{-1}$ & 405 \\
poly(AAm-co-Ru(bpy) $\left.)_{3}\right)$ & $-4.9 \times 10^{-3}$ & 3.87 & $8.2 \times 10^{-1}$ & 428 \\
poly(NIPAAm-co-Ru(bpy) $\left.)_{3}\right)$ & $-7.9 \times 10^{-3}$ & 2.86 & $8.2 \times 10^{-1}$ & 486 \\
\hline
\end{tabular}

The excitation wavelength was $490 \mathrm{~nm}$. The residual errors were less than 1.0.

All the luminescence decay curves of the suspensions were fitted by a double-exponential model, which is expressed as

$F(t)=A+B_{1} \exp \left(-\frac{t}{\tau_{1}}\right)+B_{2} \exp \left(-\frac{t}{\tau_{2}}\right)$

$\left(F(t)\right.$ : luminescence intensity at time $t ; A, B_{1}$ and $B_{2}$ are pre-exponential factors; $T_{1}$ and $T_{2}$ are decay time). To obtain the fitting curves with small residuals $\left(\chi^{2}<1.0\right)$, the luminescence lifetimes were fitted with the double-exponential model. The decay was fitted to a minor longlived component $\left(\tau_{1}\right)$ and a major short-lived component $\left(\tau_{2}\right)$. Considering that the values of the $B_{1}$ are substantially smaller than the $B_{2}$, the values of $\tau_{2}$ are effective. 
a

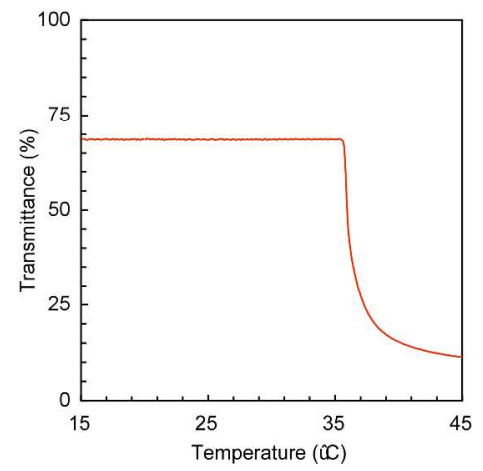

b

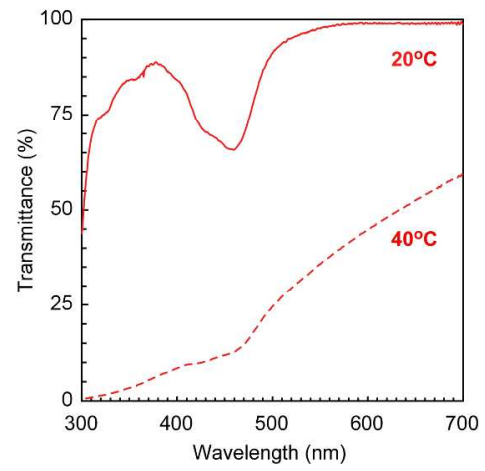

Figure S2. a) Temperature dependence of transmittance for the poly(NIPAAm-co-Ru(bpy $\left.)_{3}\right)$ solution. $\lambda=460 \mathrm{~nm}$. b) Transmittance of the poly(NIPAAm-co-Ru(bpy) $\left.)_{3}\right)$ solution as a function of wavelength at $20{ }^{\circ} \mathrm{C}$ and $40{ }^{\circ} \mathrm{C}$. [EDTA $]_{0}=50 \mathrm{mM}$.

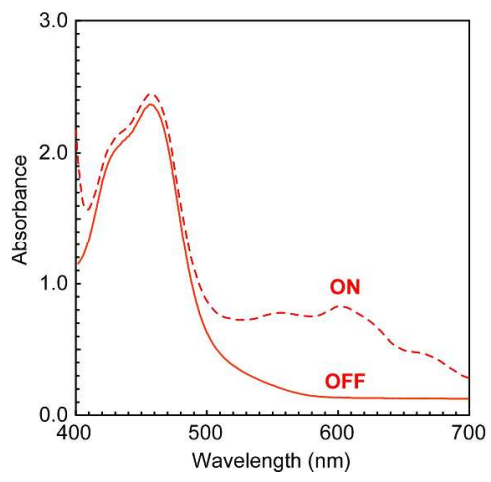

Figure S3. Change in absorption spectra for the $\mathrm{G}$ system at $25^{\circ} \mathrm{C}$ and light irradiation. [EDTA] $]_{0}$ $=50 \mathrm{mM} ;\left[\mathrm{MV}^{2+}\right]_{0}=5.0 \mathrm{mM}$.

0

30

G

L

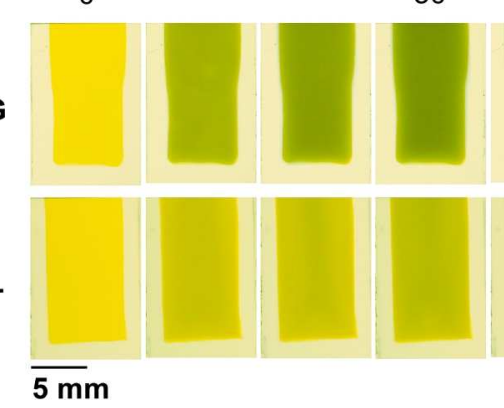

60
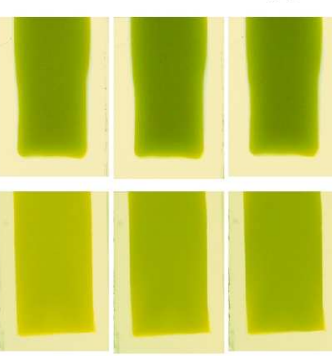

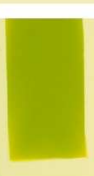

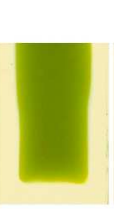

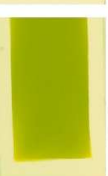

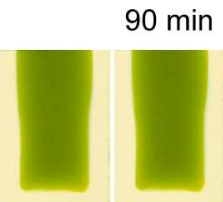
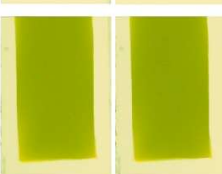

Figure S4. Visible light irradiation for the poly(NIPAAm-co-Ru(bpy) $)_{3}$ ) gel sheet containing EDTA and $\mathrm{MV}^{2+}$ (G system), and for the mixture of EDTA, Ru(bpy) ${ }_{3}{ }^{2+}$, and $\mathrm{MV}^{2+}$ (L system). 


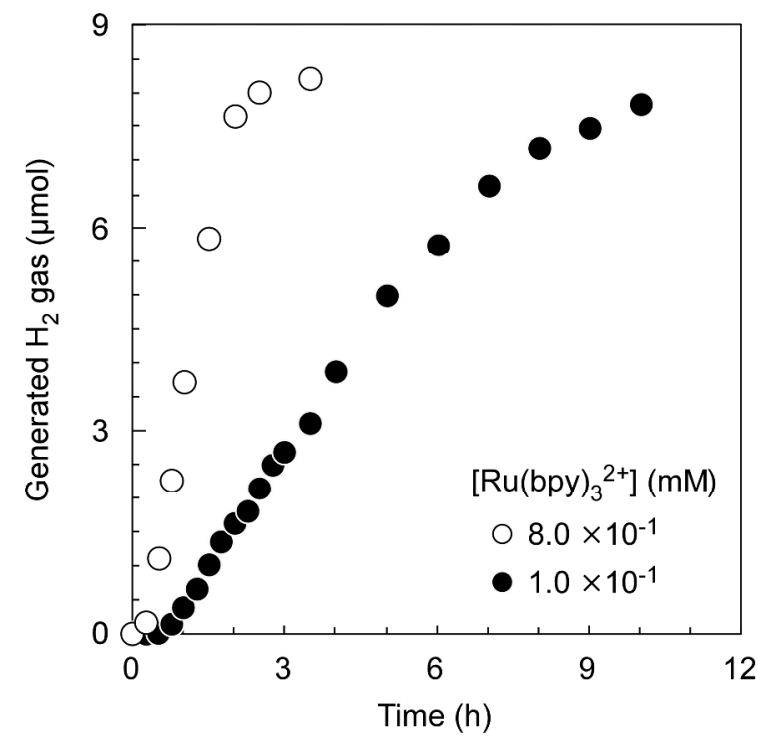

Figure S5. $\mathrm{H}_{2}$ generation for the microgel suspension using poly(NIPAAm-co-Ru(bpy)3) gel containing Pt NPs at $20{ }^{\circ} \mathrm{C}$ under air atmosphere and light irradiation. The total volume of the mixture: $3.0 \mathrm{~mL}$. The total amounts of gel particles: $20 \mathrm{mg}$. [EDTA $]_{0}=50 \mathrm{mM}$; $\left[\mathrm{MV}_{2+}\right]_{0}=5.0$ $\mathrm{mM}$.

With an increase in the $\mathrm{H}_{2}$ generating rate, the duration time for the $\mathrm{H}_{2}$ generation became short. The duration time depends on the $\mathrm{H}_{2}$ generating rate because the reaction stops when the sacrificial reducer EDTA is consumed. 